Article

\title{
Simultaneous Estimation of Twenty Eight Phenolic Compounds by a Novel and Expeditious Method Developed on Quaternary Ultra-Performance Liquid Chromatography System with a Photodiode Array Detector
}

\author{
Shiwani Mandhania ${ }^{1, *\left(\mathbb{C}, \text { Ajay Pal }^{2} \text { and Vinod Saharan }\right.}{ }^{3}$ \\ 1 Cotton Biochemistry Laboratory, Cotton Section, Department of Genetics and Plant Breeding, CCS Haryana \\ Agricultural University, Hisar, Haryana 125 004, India \\ 2 Department of Biochemistry, College of Basic Sciences and Humanities, \\ CCS Haryana Agricultural University, Hisar, Haryana 125 004, India; ajaydrdo@rediffmail.com \\ 3 Nano Research Facility Lab, Department of Molecular Biology and Biotechnology, Maharana Pratap \\ University of Agriculture and Technology, Udaipur, Rajasthan 313 001, India; vinodsaharan@gmail.com \\ * Correspondence: smbiochem@gmail.com
}

Received: 18 November 2019; Accepted: 16 December 2019; Published: 18 December 2019

\begin{abstract}
Plant secondary metabolites including phenolics and flavonoidsare synthesized through phenylpropanoid and phenylpropanoid-acetate pathways and significantly contribute against adverse effect of abiotic and biotic stresses. Herein, we present the development and execution of a novel and expeditious ultra-performance liquid chromatographic-photodiode array (UPLC-PDA) method for qualitative and quantitative analysis of 28 phenolic compounds comprising of flavonoids, phenolic acids, aldehydes and alcohols. The method is able to separate phenolic compounds in just $17 \mathrm{~min}$ with the separation of isobaric species such as 3,4 dihydroxybenzoic acid and 3,5 dihydroxy benzoic acid; quercetin and taxifolin. Linear curves concentrations ranged from $6-18 \mu \mathrm{g} / \mathrm{mL}$ (3,5 dihydroxy benzoic acid), 4-12 $\mu \mathrm{g} / \mathrm{mL}$ (catechin and salicylic acid) and 2-6 $\mu \mathrm{g} / \mathrm{mL}$ for rest of the compounds and correlation coefficients were $>0.994$. The limit of detection (LOD) varied from $0.04-0.45 \mu \mathrm{g} / \mathrm{mL}$. Cotton root samples were used to assess the method in terms of recovery efficiency $(85-120 \%)$, precision $(0.12-4.09 \%)$ and intermediate precision $(0.32-4.0 \%)$.Phenolics and flavonoidsin root samples of healthy and diseased plants as well as leaf samples of healthy plants were successfully quantified using this novel method without an expensive Mass Spectrometer.
\end{abstract}

Keywords: UPLC; PDA; Phenolics; Flavonoids; Cotton; Root rot

\section{Introduction}

Cotton, also known as "white gold" is grown as a fiber and food crop all over the world. Amongst the major cultivated species of cotton, Gossypium hirsutum L. significantly contributes to total lint cotton production [1,2]. India ranks first in terms of area of cultivation but lags behind in productivity, which is severely affected by various abiotic and biotic stresses [3]. Cotton plants are attacked by 1326 species of insects and dozens of pathogens [4]. Among all, cotton leaf curl virus and $R$. solani severely affect the productivity and accounts for up to $40 \%$ reduction in yield $[5,6]$. The infection and infestation of plants lead to activation of protein inhibitors and induction of defense mechanisms including the release of secondary metabolites like phenolic compounds $[7,8]$. Phenylalanine and some phenolic compounds are derived from phosphoenol phosphate and erythrose-4-phosphate through the shikimate pathway. The former is converted into cinnamic acid which acts as a precursor for biosynthesis of 
phenolic compounds. Cinnamic acid via coumaroyl-CoA is routed through the phenylpropanoid pathway for synthesis of additional phenolics and lignin polymerization. Alternatively, it is routed through a phenylpropanoid-acetate pathway for the biosynthesis of flavonoids and isoflavonoids (Figure 1). The diversity of phenolics with acid, aldehyde or alcohol groups and their acylation and glycosylation make the process of separation and quantification difficult in complex plant matrices. Currently, several methodologies are in use for the analysis of phenolic compounds in plant systems. Amongst them, spectrophotometric-based Folin-Denis and differential $\mathrm{pH}$ assays are preferably used for quantification of total phenolics and anthocyanins, respectively [8]. However, these methods lack the specificity as well as selectivity which lead to over or underestimation of phenolic content [9]. So, there is an urgentnecessity to quantify the differential phenolics using a reliable, rapid and sensitive analytical technique to provide raw screened breeding materials to plant pathologists and breeders to boost pathway discovery as well as metabolic engineering $[10,11]$. Amongst the various analytical techniques being currently practiced for separation and quantification of phenolics, a high-performance liquid chromatography-photodiode array detector (HPLC-PDA) using a reverse-phase C18 column is commonly applied [12-15]. Some free and bound phenolic acids as well as flavonoids, for example, have been simultaneously quantified in food and plant samples using this technique $[16,17]$. The chemical diversity and complexity of these compounds have been analyzed through an untargeted approach using high-resolution mass spectrometry [18-21], whereas triple quadruple has been used to specifically quantify the targeted compounds. However, only phenolics or flavonoids have been considered at a time in most of the studies [22,23]. Very few studies have focused on the simultaneous estimation of all types of phenolic compounds including acid, aldehyde and flavonoids [11,24,25]. Moreover, none of the studies have accomplished the simultaneous separation and quantification of phenolics, phenolic acid, derivatives of hydroxycinnamic acid and flavonoids which are crucial for lignin biosynthesis.

The present work illustrates the development of a method to separate and quantify 28 phenolic compounds of different chemistry using ultra-performance liquid chromatography system equipped with a photodiode array detector (UPLC-PDA). The study achieved a secondary metabolites chromatographic resolution including isobaric species in less than $19 \mathrm{~min}$. The developed method is fully validated for simultaneous identification of 28 phenolic compounds to characterize leaf and root samples of cotton.

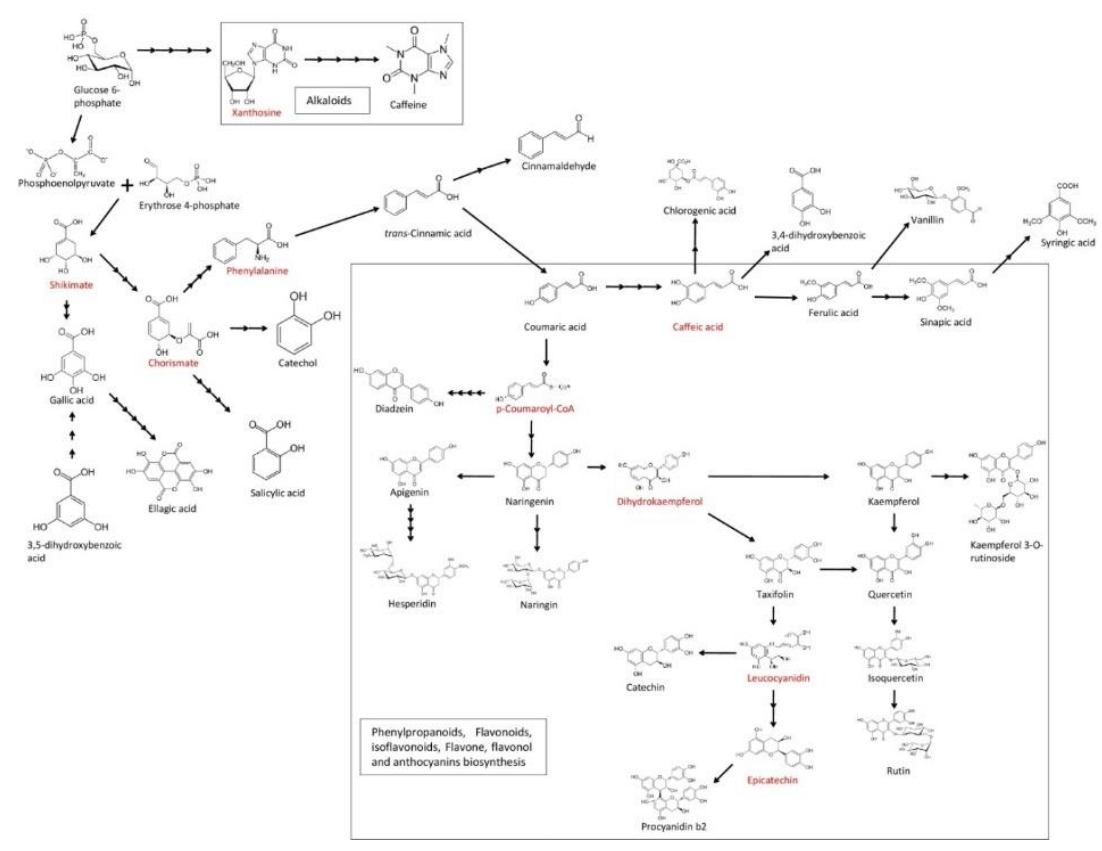

Figure 1. Schematic diagram of the phenylpropanoid biosynthetic pathway. 


\section{Materials and Methods}

\subsection{Chemicals}

Acetonitrile and formic acid of HPLC grade were supplied by Merck-Sigma (Germany). Ultrapure water was obtained from Millipore Q 8 System (Millipore, MA, USA).

\subsection{Standards}

Trans-cinnamic acid; 3,5 dihydroxybenzoicacid (3,5 DHBA); chlorogenic acid; catechin; 2-methoxycinnmaldehyde (2-MC); ellagic acid; ( \pm ) -naringenin; naringin; procyanidin B2; trans-sinapic acid; taxifolin were procured from Sigma Aldrich (St Louis, MO, USA). Rutin hydrate; syringic acid; kaempferol; isoquercetin; kaempferol-3-O- $\beta$ rutinoside (K-3-O- $\beta \mathrm{R})$; daidzein were obtained from Sigma (St Louis, MO, USA). Gallic acid; caffeine; vanillin; salicyclic acid; 3,4 dihyroxybenzoic acid (3,4 DHBA); trans-ferulic acid; trans- $p$-coumaric acid were procured from Fluka (St Louis, MO, USA). Catechol; hesperdin; apigenin; quercetin dihydrate were purchased from Alfa Acer, Thermo Fisher Scientific (USA). Stock solutions (2000 ppm) of individual compound were prepared in methanol. Two separate intermediate solutions of all the standards were prepared. One was used for the estimation of LOQ (limit of quantification) and LOD (limit of detection) while the other for recovery and linearity by diluting in methanol as depicted in Table 1. The working solution was further prepared by diluting in mobile phase A and B in the ratio 80:20.

Table 1. Concentrations of intermediate solutions and $\lambda_{\max }$ of standards.

\begin{tabular}{|c|c|c|c|c|}
\hline Sr. No. & Compound & $\begin{array}{l}\text { LOD and LOQ } \\
(\mu \mathrm{g} / \mathrm{mL})\end{array}$ & $\begin{array}{c}\text { Linearity and } \\
\text { Recovery }(\mu \mathrm{g} / \mathrm{mL})\end{array}$ & $\lambda_{\max }$ \\
\hline 1 & Gallic acid & 8.0 & 40.0 & 214.7 \\
\hline 2 & 3,5DHBA & 40.0 & 120.0 & 209.7 \\
\hline 3 & $3,4 \mathrm{DHBA}$ & 15.0 & 40.0 & 209.7 \\
\hline 4 & Catechol & 20.0 & 40.0 & 209.7 \\
\hline 5 & Chlorogenic acid & 20.0 & 40.0 & 324.9 \\
\hline 6 & Catechin & 45.0 & 80.0 & 209.7 \\
\hline 7 & Caffeine & 8.0 & 40.0 & 209.7 \\
\hline 8 & Syringic acid & 10.0 & 40.0 & 216.8 \\
\hline 9 & ProcyanidinB2 & 30.0 & 40.0 & 209.7 \\
\hline 10 & Vanillin & 20.0 & 40.0 & 229.7 \\
\hline 11 & Trans- $p$-coumaric acid & 8.0 & 40.0 & 309.3 \\
\hline 12 & Salicylic acid & 40.0 & 80.0 & 209.7 \\
\hline 13 & Trans-ferulic acid & 15.0 & 40.0 & 321.3 \\
\hline 14 & Trans-sinapicacid & 40.0 & 40.0 & 322,5 \\
\hline 15 & Taxifolin & 8.0 & 40.0 & 209.7 \\
\hline 16 & Ellagic acid & 40.0 & 40.0 & 253.4 \\
\hline 17 & Rutinhydrate & 40.0 & 40.0 & 209.7 \\
\hline 18 & Isoquercetin & 30.0 & 40.0 & 209.7 \\
\hline 19 & K-3-O- $\beta R$ & 30.0 & 40.0 & 209.7 \\
\hline 20 & Naringin & 15.0 & 40.0 & 213.2 \\
\hline 21 & Hesperidin & 15.0 & 40.0 & 209.7 \\
\hline 22 & Daidzein & 15.0 & 40.0 & 248.7 \\
\hline 23 & Trans-cinnamic acid & 8.0 & 40.0 & 277.1 \\
\hline 24 & Quercetin dihydrate & 40.0 & 40.0 & 209.7 \\
\hline 25 & Naringenin & 15.0 & 40.0 & 212.0 \\
\hline 26 & Apigenin & 15.0 & 40.0 & 209.7 \\
\hline 27 & Kaempferol & 20.0 & 40.0 & 209.7 \\
\hline 28 & 2-MC & 4.0 & 40.0 & 286.7 \\
\hline
\end{tabular}




\subsection{Samples}

Root and leaf samples of cotton plant were taken from the healthy and sick plot area (field area maintained for growth and maintenance of Rhizoctonia culture for screening of cotton root rot tolerant genotypes) of cotton research farm area, CCS Haryana Agricultural University, Hisar, in Kharif season 2018. Root samples collected from healthy and sick plot area served as sample D (healthy) and C (diseased), respectively while leaf sample from healthy plant served as sample L. Phenolic compounds were extracted from root $(0.2 \mathrm{~g})$ and leaf $(2.0 \mathrm{~g})$ samples following the method of Adomand Liu [26]. The extracted samples were further cleaned using Sep-Pak C18 and Oasis HLB 6CC cartridges (Waters MA, USA) as described earlier [27] with minor modifications. The samples were finally dissolved in methanol and filtered through nylon 0.22-micron syringe filters.

\subsection{Instrumentation}

Phenolic compounds were analyzed using quaternary ultra-performance liquid chromatography (UPLC) equipped with a photodiode array detector (PDA) (H-Class Acquity, Waters, Milford, MA, USA). The BEH HSS C 18 column having $1.7 \mu \mathrm{m}$ particle size and $2.1 \mathrm{~mm}$ inlet diameter was used to resolve 28 phenolics. To achieve the resolution, different gradient systems with varied concentrations of additive (formic acid) was examined. Acetonitrile (solvent B) and $0.01 \%$ formic acid in water (solvent A) with a flow rate of $600 \mu \mathrm{L} /$ minute proved best for resolution of phenolic compounds and isomers of phenolics and flavonoids under study (data not shown) at $35^{\circ} \mathrm{C}$ column temperature. The optimized gradient elution began with $95 \%$ (solvent $\mathrm{A}$ ) and adjusted to $85 \%$ at $8.30 \mathrm{~min} ; 80 \%$ (solvent $\mathrm{A}$ ) and $20 \%$ (solvent B) at $10.60 \mathrm{~min} ; 70 \%$ (solvent $\mathrm{A}$ ) and $30 \%$ (solvent $\mathrm{B}$ ) at $12.90 \mathrm{~min} ; 50 \%$ of both solvents (A and B) at $13.90 \mathrm{~min}$ and continued up to $14.60 \mathrm{~min}$. The column was conditioned with an initial injection condition of $95 \%$ (A) and 5\% (B) from 14.65 to $17.00 \mathrm{~min}$ and next injection was delayed by $2.0 \mathrm{~min}$. The absorbance spectrum of standards was obtained in the range 190-400 $\mathrm{nm}$ using a PDA detector to find wavelength maxima $\left(\lambda_{\max }\right)$. The $\lambda_{\max }$ of individual 28 phenolic compounds in mixed solution is given in Table 1. Based on peak intensity measured, a wavelength of $278 \mathrm{~nm}$ was chosen for detection of all the phenolic compounds.

\subsection{Analytical Method Validation}

The characteristics of method performance were established with assays of blanks, standards, selectivity and system suitability, linearity, recovery, precision, intermediate precision, LOD and LOQ at $5 \%$ significance level.

\subsubsection{Selectivity and System Suitability}

Peak purities of 28 standards and a root sample of cotton spiked with these standards were determined by analyzing the PDA spectra at different peak points. System suitability was gauged by examining the consequences from USP criteria obtained at $100 \%$ level of concentrations with six injections.

\subsubsection{Linearity}

Five variable concentrations (50, 75, 100, 125 and 150\%) including one base concentration (100\%) of 28 standards were prepared from mixed solution. The base concentration was $8 \mu \mathrm{g} / \mathrm{mL}$ for catechin and salicylic acid; $12 \mu \mathrm{g} / \mathrm{mL}$ for 3,5 dihydroxy benzoic acid and $4 \mu \mathrm{g} / \mathrm{mL}$ for rest of the phenolic compounds. Calibration curves were made from these concentrations, analyzed in duplicate over UPLC-PDA and regression analysis was computed through built-in Empower 3 software. 


\subsubsection{Precision and Accuracy}

Analysis of duplicate injections of two different samples in two replications was carried out on same and different days to check the intra and inter-day precision (intermediate precision). Relative standard deviation (RSD) of the values expressed the precision of method.

For estimation of recovery, unfiltered sample was spiked with three concentrations (75, 100, $125 \%$ ) of standards. The developed method was applied to analyze two independent samples at each concentration and percent recovery was expressed as-

$$
\text { [Calculated concentration/Theoretical concentration] } \times 100
$$

\subsubsection{Limit of Quantification (LOQ) and Limit of Detection (LOD)}

Serial dilutions of standards were prepared by diluting in mobile phase A and B in the ratio 80:20 and used for estimation of LOD. The signal (s) to noise (n) ratios of 3 and 10 were used to express LOD and LOQ, respectively.

\section{Results and Discussion}

\subsection{UPLC Method Development}

HPLC methods used in cotton plant and food [12,15] were adopted for the maturation of present analytical method. The optimized UPLC mobile phase consists of (A) $0.01 \%$ formic acid in Milli Q water ( $\mathrm{pH}=3.34)$ : (B) ACN system on a Waters column with an optimal gradient of $17 \mathrm{~min}$. A total of 28 standards were identified to quantify the phenolic compounds in samples (Figure 2).

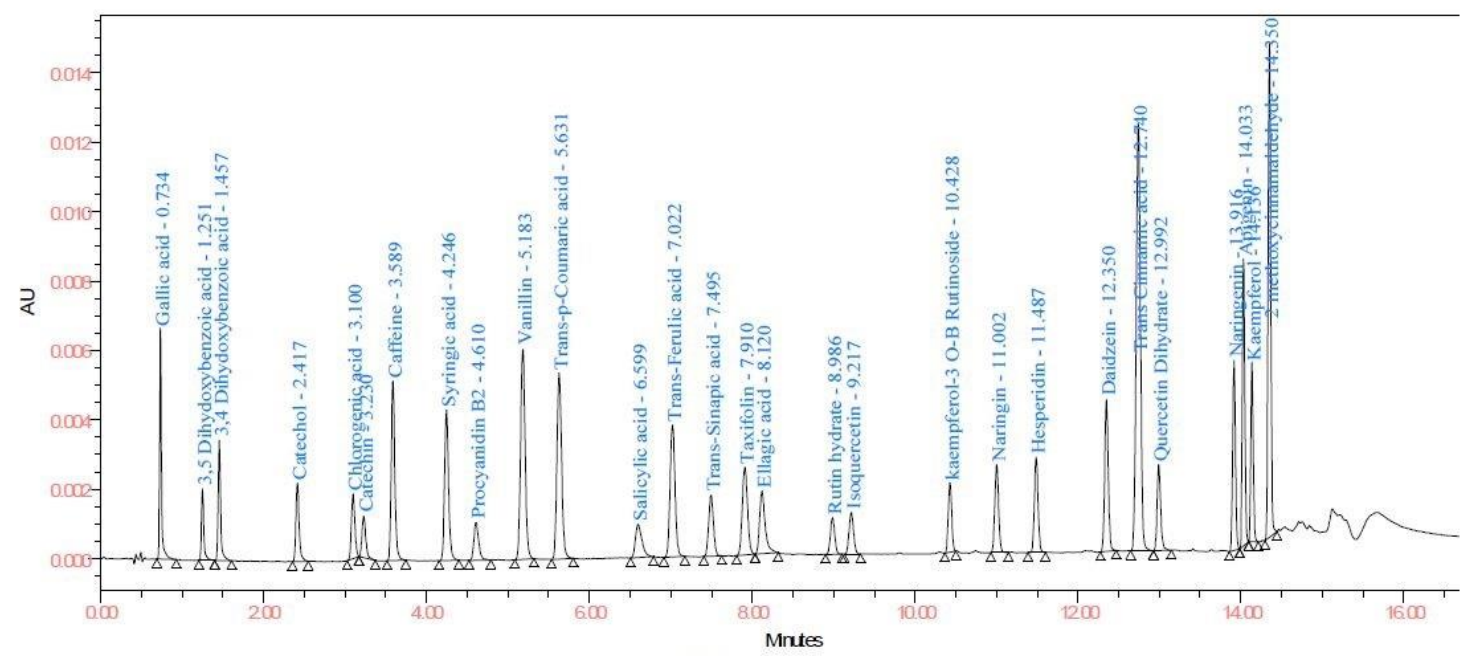

Figure 2. Chromatogram of standard phenolics at $278 \mathrm{~nm}$.

\subsection{Analytical Method Validation}

\subsubsection{Selectivity and System Suitability}

Identification and quantification of 28 peaks from cotton root extract spiked with standard phenolics and related reference standards were reviewed (Supplementary Material A 1-3) and results show that other compounds did not co-elute (Tables 2 and 3). 
Table 2. Peak purities of standards, spiked sample C and D for assessment of system suitability of method.

\begin{tabular}{|c|c|c|c|c|c|c|c|c|c|c|c|c|c|}
\hline \multirow{2}{*}{ Sr. No. } & \multirow{2}{*}{ Compound } & \multicolumn{4}{|c|}{ Peak Purity of Standard } & \multicolumn{4}{|c|}{ Peak purity of Spiked Sample C } & \multicolumn{4}{|c|}{ Peak Purity of Spiked Sample D } \\
\hline & & RT & Area & $\begin{array}{l}\text { Purity } \\
\text { Angle }\end{array}$ & $\begin{array}{l}\text { Purity } \\
\text { Threshold }\end{array}$ & RT & Area & $\begin{array}{l}\text { Purity } \\
\text { Angle }\end{array}$ & $\begin{array}{c}\text { Purity } \\
\text { Threshold }\end{array}$ & RT & Area & $\begin{array}{l}\text { Purity } \\
\text { Angle }\end{array}$ & $\begin{array}{c}\text { Purity } \\
\text { Threshold }\end{array}$ \\
\hline 1 & Gallic acid & 0.739 & 11215 & 0.719 & 2.660 & 0.739 & 10690 & 0.855 & 2.754 & 0.738 & 10905 & 0.758 & 2.798 \\
\hline 2 & 3,5 DHBA & 1.256 & 4009 & 0.230 & 1.966 & 1.258 & 4047 & 0.189 & 1.983 & 1.258 & 4119 & 0.249 & 2.058 \\
\hline 3 & $3,4 \mathrm{DHBA}$ & 1.460 & 7211 & 0.436 & 2.288 & 1.463 & 7402 & 0.499 & 2.557 & 1.462 & 7332 & 0.454 & 2.525 \\
\hline 4 & Catechol & 2.421 & 6245 & 2.004 & 4.969 & 2.412 & 6520 & 3.355 & 6.878 & 2.413 & 6597 & 4.331 & 6.885 \\
\hline 5 & Chlorogenic acid & 3.100 & 5562 & 0.866 & 2.316 & 3.072 & 5758 & 1.054 & 2.586 & 3.079 & 5862 & 0.772 & 2.501 \\
\hline 6 & Catechin & 3.232 & 3938 & 8.175 & 7.158 & 3.204 & 4306 & 9.411 & 9.754 & 3.211 & 4083 & 6.609 & 8.747 \\
\hline 7 & Caffeine & 3.589 & 15622 & 0.648 & 2.712 & 3.552 & 16310 & 2.094 & 3.415 & 3.558 & 16204 & 1.370 & 3.516 \\
\hline 8 & Syringic acid & 4.246 & 14732 & 0.635 & 2.705 & 4.208 & 15648 & 0.836 & 3.268 & 4.215 & 15669 & 0.761 & 3.246 \\
\hline 9 & Procyanidin B2 & 4.609 & 4456 & 2.879 & 6.805 & 4.567 & 4818 & 4.279 & 10.237 & 4.574 & 4650 & 4.204 & 10.033 \\
\hline 10 & Vanillin & 5.186 & 23682 & 0.315 & 2.103 & 5.151 & 25149 & 0.370 & 2.301 & 5.158 & 25133 & 0.361 & 2.270 \\
\hline 11 & Trans- $p$-coumaric acid & 5.634 & 21509 & 0.263 & 1.957 & 5.601 & 22689 & 0.285 & 2.049 & 5.609 & 22487 & 0.234 & 2.026 \\
\hline 12 & Salicylic acid & 6.605 & 5213 & 0.905 & 3.261 & 6.575 & 5581 & 0.950 & 3.454 & 6.582 & 5444 & 0.901 & 3.465 \\
\hline 13 & Trans-ferulic acid & 7.028 & 16178 & 0.258 & 1.991 & 6.996 & 18347 & 0.213 & 1.984 & 7.005 & 17770 & 0.257 & 2.063 \\
\hline 14 & Trans-sinapicacid & 7.501 & 7192 & 0.276 & 2.013 & 7.468 & 7315 & 0.291 & 2.127 & 7.477 & 7289 & 0.278 & 2.125 \\
\hline 15 & Taxifolin & 7.918 & 11366 & 0.727 & 2.826 & 7.884 & 11727 & 0.880 & 3.072 & 7.894 & 11904 & 0.776 & 3.183 \\
\hline 16 & Ellagic acid & 8.130 & 8676 & 2.871 & 2.903 & 8.095 & 8598 & 3.021 & 3.438 & 8.106 & 8621 & 3.082 & 3.410 \\
\hline 17 & Rutin hydrate & 8.997 & 3836 & 0.587 & 2.683 & 8.951 & 4099 & 0.666 & 2.972 & 8.959 & 3865 & 0.533 & 2.832 \\
\hline 18 & Isoquercetin & 9.228 & 4574 & 0.647 & 2.679 & 9.182 & 4668 & 0.550 & 2.693 & 9.189 & 4696 & 0.550 & 2.797 \\
\hline 19 & $\mathrm{~K}-3-\mathrm{O}-\beta \mathrm{R}$ & 10.437 & 6280 & 0.597 & 2.337 & 10.399 & 6003 & 0.373 & 2.285 & 10.402 & 6071 & 0.361 & 2.317 \\
\hline 20 & Naringin & 11.009 & 8451 & 1.786 & 3.553 & 10.978 & 8729 & 1.897 & 4.113 & 10.978 & 8811 & 1.975 & 4.261 \\
\hline 21 & Hesperidin & 11.495 & 8761 & 1.077 & 3.313 & 11.466 & 9162 & 1.266 & 3.886 & 11.465 & 9140 & 1.284 & 3.776 \\
\hline 22 & Daidzein & 12.358 & 13358 & 0.395 & 2.126 & 12.332 & 14063 & 0.499 & 2.215 & 12.333 & 13816 & 0.404 & 2.205 \\
\hline 23 & Trans-cinnamic acid & 12.749 & 45275 & 0.255 & 1.950 & 12.719 & 47365 & 0.384 & 2.075 & 12.720 & 47448 & 0.295 & 2.104 \\
\hline 24 & Quercetin dihydrate & 12.999 & 7423 & 0.359 & 2.056 & 12.977 & 8034 & 1.629 & 2.095 & 12.977 & 8022 & 0.957 & 2.215 \\
\hline 25 & Naringenin & 13.925 & 13065 & 0.316 & 2.167 & 13.905 & 13839 & 0.434 & 2.314 & 13.905 & 13835 & 0.336 & 2.357 \\
\hline 26 & Apigenin & 14.041 & 17240 & 0.130 & 1.699 & 14.024 & 18187 & 0.195 & 1.726 & 14.024 & 18054 & 0.155 & 1.741 \\
\hline 27 & Kaempferol & 14.143 & 10491 & 0.223 & 1.756 & 14.128 & 10972 & 0.280 & 1.784 & 14.128 & 10976 & 0.231 & 1.798 \\
\hline 28 & 2-MC & 14.358 & 32624 & 0.549 & 1.762 & 14.340 & 33852 & 0.404 & 1.807 & 14.340 & 33499 & 0.255 & 1.787 \\
\hline
\end{tabular}


Table 3. System suitability parameters, correlation coefficients, LOD and LOQ of the method.

\begin{tabular}{|c|c|c|c|c|c|c|c|c|c|c|c|c|}
\hline \multirow{2}{*}{ Sr. No. } & \multirow{2}{*}{ Compound } & \multicolumn{7}{|c|}{ System Suitability } & \multirow{2}{*}{$\begin{array}{l}\text { Correlation } \\
\text { Coefficient }\end{array}$} & \multirow{2}{*}{$\begin{array}{c}\text { Linearity } \\
\text { Range } \\
(\mu \mathrm{g} / \mathrm{mL})\end{array}$} & \multirow{2}{*}{$\begin{array}{l}\text { LOD } \\
(\mu \mathrm{g} / \mathrm{mL})\end{array}$} & \multirow{2}{*}{$\underset{(\mu \mathrm{g} / \mathrm{mL})}{\mathrm{LOQ}}$} \\
\hline & & RT & RSD & Area & RSD & $\begin{array}{c}\text { USP } \\
\text { Resolution }\end{array}$ & $\begin{array}{c}\text { USP } \\
\text { Tailing }\end{array}$ & $\begin{array}{l}\text { USP Plate } \\
\text { Count }\end{array}$ & & & & \\
\hline 1 & Gallic acid & 0.739 & 0.2 & 11215 & 0.91 & & 1.43 & 5384 & 0.998 & $2-6$ & 0.080 & 0.264 \\
\hline 2 & 3,5 DHBA & 1.256 & 0.3 & 4009 & 1.44 & 11.25 & 1.46 & 10198 & 0.995 & $6-18$ & 0.400 & 1.320 \\
\hline 3 & 3,4 DHBA & 1.460 & 0.2 & 7211 & 1.23 & 3.91 & 1.40 & 12274 & 0.996 & $2-6$ & 0.150 & 0.495 \\
\hline 4 & Catechol & 2.421 & 0.0 & 6245 & 1.04 & 15.42 & 1.27 & 19593 & 0.995 & $2-6$ & 0.200 & 0.660 \\
\hline 5 & Chlorogenic acid & 3.100 & 0.1 & 5562 & 0.37 & 8.97 & 1.08 & 24397 & 0.996 & $2-6$ & 0.200 & 0.660 \\
\hline 6 & Catechin & 3.232 & 0.1 & 3938 & 0.53 & 1.57 & 1.46 & 23646 & 0.997 & $4-12$ & 0.450 & 1.485 \\
\hline 7 & Caffeine & 3.589 & 0.2 & 15622 & 0.55 & 4.31 & 1.21 & 34002 & 0.997 & $2-6$ & 0.080 & 0.264 \\
\hline 8 & Syringic acid & 4.246 & 0.2 & 14732 & 0.29 & 7.74 & 1.19 & 37086 & 0.998 & $2-6$ & 0.100 & 0.330 \\
\hline 9 & Procyanidin B2 & 4.609 & 0.2 & 4456 & 2.26 & 3.63 & 1.26 & 29671 & 0.998 & 2- 6 & 0.300 & 0.990 \\
\hline 10 & Vanillin & 5.186 & 0.1 & 23682 & 0.31 & 5.39 & 1.11 & 41111 & 0.997 & $2-6$ & 0.200 & 0.660 \\
\hline 11 & Trans- $p$-coumaric acid & 5.634 & 0.1 & 21509 & 0.31 & 4.25 & 1.15 & 46333 & 0.997 & $2-6$ & 0.080 & 0.264 \\
\hline 12 & Salicylic acid & 6.605 & 0.1 & 5213 & 1.67 & 7.62 & 1.41 & 32991 & 0.994 & $4-12$ & 0.400 & 1.320 \\
\hline 13 & Trans-ferulic Acid & 7.028 & 0.1 & 16178 & 0.46 & 3.23 & 1.10 & 63206 & 0.997 & $2-6$ & 0.150 & 0.495 \\
\hline 14 & Trans-sinapic acid & 7.501 & 0.1 & 7192 & 0.18 & 4.25 & 1.08 & 76938 & 0.996 & $2-6$ & 0.400 & 1.320 \\
\hline 15 & Taxifolin & 7.918 & 0.1 & 11366 & 0.44 & 3.59 & 1.08 & 69063 & 0.996 & $2-6$ & 0.080 & 0.264 \\
\hline 16 & Ellagic acid & 8.130 & 0.1 & 8676 & 2.07 & 1.72 & 1.63 & 74926 & 0.995 & $2-6$ & 0.400 & 1.320 \\
\hline 17 & Rutin hydrate & 8.997 & 0.1 & 3836 & 1.22 & 7.80 & 1.09 & 133785 & 0.997 & $2-6$ & 0.400 & 1.320 \\
\hline 18 & Isoquercetin & 9.228 & 0.1 & 4574 & 0.61 & 2.26 & 1.11 & 127943 & 0.996 & $2-6$ & 0.300 & 0.990 \\
\hline 19 & $\mathrm{~K}-3-\mathrm{O}-\beta \mathrm{R}$ & 10.437 & 0.0 & 6280 & 3.71 & 12.98 & 1.15 & 269816 & 0.997 & 2- 6 & 0.300 & 0.990 \\
\hline 20 & Naringin & 11.009 & 0.0 & 8451 & 0.62 & 6.76 & 1.05 & 258318 & 0.998 & $2-6$ & 0.150 & 0.495 \\
\hline 21 & Hesperidin & 11.495 & 0.0 & 8761 & 0.17 & 5.59 & 1.09 & 297168 & 0.998 & $2-6$ & 0.150 & 0.495 \\
\hline 22 & Daidzein & 12.358 & 0.0 & 13358 & 0.43 & 10.33 & 1.10 & 378827 & 0.997 & $2-6$ & 0.150 & 0.495 \\
\hline 23 & Trans-cinnamic acid & 12.749 & 0.0 & 45275 & 0.13 & 4.33 & 1.05 & 274170 & 0.997 & $2-6$ & 0.080 & 0.264 \\
\hline 24 & Quercetin dehydrate & 12.999 & 0.0 & 7423 & 0.28 & 2.79 & 1.24 & 430037 & 0.996 & $2-6$ & 0.400 & 1.320 \\
\hline 25 & Naringenin & 13.925 & 0.0 & 13065 & 0.24 & 12.73 & 1.07 & 764867 & 0.997 & $2-6$ & 0.150 & 0.495 \\
\hline 26 & Apigenin & 14.041 & 0.0 & 17240 & 0.27 & 1.90 & 1.13 & 990979 & 0.994 & $2-6$ & 0.150 & 0.495 \\
\hline 27 & Kaempferol & 14.143 & 0.0 & 10491 & 0.21 & 1.81 & 1.18 & 1050030 & 0.996 & $2-6$ & 0.200 & 0.660 \\
\hline 28 & $2-\mathrm{MC}$ & 14.358 & 0.0 & 32624 & 0.47 & 3.66 & 1.05 & 899325 & 0.997 & $2-6$ & 0.040 & 0.132 \\
\hline
\end{tabular}


Elution of $p$-coumaric acid, ferulic acid and sinapic acid with major phenolic acids usually requires 40-50 min by HPLC [28]. Mattila and Kumpulainen [16], however, reduced time to 27 min but ferulic and sinapic acids could not be resolved. But, in the present method system suitability indicates consistent chromatographic conditions like USP tailing $<1.5$; USP resolution $>1.5$; selectivity $>1$; number of theoretical plates $>7000$, according to the US FDA (FDA, 1994) within 17 min.

\subsubsection{Linearity, LOQ and LOD}

Linear curves regression formula and correlation coefficients of standards are presented in Table 3. Results indicate that linearity of all 28 standards varies from 0.994 to 0.998 . Fracassetti et al. [29] prepared calibration curves of catechin and caffeic acid in the ranges from 0.5 to $80 \mu \mathrm{g} / \mathrm{mL}$ and 0.5 to $50 \mu \mathrm{g} / \mathrm{mL}$, respectively for establishment of a UPLC method. In the present method, linear model was computed from the calibration curve (area versus amount, which fulfils the requirement of homoscedasticity (Supplementary Material B 1-28).

The LOD was $0.04-0.450 \mu \mathrm{g} / \mathrm{ml}$ on the foundation of $\mathrm{s} / \mathrm{n}$ ratio of 3 and the LOQ was 3.3 times of LOD (Table 3, Supplementary Material C 1-2). Our study corroborates with the findings of Dias et al. [30], Escarpa and González [31].

\subsubsection{Accuracy and Precision}

The results presented in Table 4 show that recovery ranges from 85 to $112 \%$ which falls within the generally accepted range of 85 to $120 \%$. Moreover, for recovery studies in quantitative analysis, there are no set official criteria and rely on analyte concentration as per AOAC guidelines [32]. The recovery of analyte at 75,100 and $125 \%$ ranged from 85 to $109 \%, 85$ to $105 \%, 87$ to $103 \%$ and 85 to $112 \%$, 87 to $105 \%, 85$ to $99 \%$ in sample C and sample D, respectively.

The repeatability RSD for precision varied from 0.12 to $2.96 \%$ and 0.62 to $4.09 \%$, whereas, intermediate precision varied from 0.32 to $2.05 \%$ and 1.04 to $4.0 \%$ in sample C and D, respectively as depicted in Table 5. The better precision of the developed UPLC method as indicated by low values of RSD could be considered of high accuracy.

Table 4. Spiked amount and recoveries in sample C and D at three concentration levels.

\begin{tabular}{|c|c|c|c|c|c|c|c|c|c|c|}
\hline \multirow{2}{*}{ Sr. No. } & \multirow{2}{*}{ Compound } & \multicolumn{3}{|c|}{ Spiked Amount $(\mu \mathrm{g} / \mathrm{mL})$} & \multicolumn{3}{|c|}{ Recovery \% (Sample C) } & \multicolumn{3}{|c|}{ Recovery \% (Sample D) } \\
\hline & & $75 \%$ & $100 \%$ & $125 \%$ & $75 \%$ & $100 \%$ & $125 \%$ & $75 \%$ & $100 \%$ & $125 \%$ \\
\hline 1 & Gallic acid & 3 & 4 & 5 & 102.06 & 96.28 & 95.53 & 100.83 & 100.54 & 93.47 \\
\hline 2 & 3,5 DHBA & 9 & 12 & 15 & 101.69 & 98.00 & 96.58 & 100.13 & 99.22 & 92.66 \\
\hline 3 & 3,4 DHBA & 3 & 4 & 5 & 105.81 & 101.53 & 99.91 & 103.01 & 102.39 & 96.35 \\
\hline 4 & Catechol & 3 & 4 & 5 & 90.29 & 88.74 & 91.24 & 104.56 & 103.04 & 96.83 \\
\hline 5 & Chlorogenic acid & 3 & 4 & 5 & 105.18 & 101.84 & 100.05 & 104.61 & 103.13 & 96.08 \\
\hline 6 & Catechin & 6 & 8 & 10 & 106.97 & 103.34 & 101.20 & 103.43 & 103.61 & 96.21 \\
\hline 7 & Caffeine & 3 & 4 & 5 & 94.11 & 93.09 & 93.62 & 111.86 & 103.63 & 96.72 \\
\hline 8 & Syringic acid & 3 & 4 & 5 & 105.63 & 101.81 & 100.67 & 103.43 & 103.10 & 96.18 \\
\hline 9 & Procyanidin B2 & 3 & 4 & 5 & 103.99 & 101.05 & 99.12 & 101.57 & 102.74 & 93.68 \\
\hline 10 & Vanillin & 3 & 4 & 5 & 92.92 & 92.19 & 92.63 & 90.54 & 93.03 & 88.13 \\
\hline 11 & Trans- $p$-coumaric acid & 3 & 4 & 5 & 93.46 & 93.29 & 93.86 & 104.07 & 103.29 & 96.98 \\
\hline 12 & Salicylic acid & 6 & 8 & 10 & 108.88 & 101.88 & 102.82 & 101.01 & 102.64 & 98.79 \\
\hline 13 & Trans-ferulic acid & 3 & 4 & 5 & 93.38 & 92.61 & 93.79 & 90.88 & 93.90 & 88.86 \\
\hline 14 & Trans-sinapic acid & 3 & 4 & 5 & 105.12 & 101.19 & 100.08 & 103.27 & 103.19 & 95.87 \\
\hline 15 & Taxifolin & 3 & 4 & 5 & 105.45 & 101.96 & 100.57 & 103.73 & 102.86 & 96.12 \\
\hline 16 & Ellagic acid & 3 & 4 & 5 & 104.55 & 100.46 & 100.02 & 102.01 & 101.39 & 94.23 \\
\hline 17 & Rutin hydrate & 3 & 4 & 5 & 106.91 & 103.03 & 102.01 & 100.60 & 102.81 & 96.45 \\
\hline 18 & Isoquercetin & 3 & 4 & 5 & 105.70 & 101.14 & 100.28 & 101.86 & 101.86 & 95.25 \\
\hline 19 & $\mathrm{~K}-3-\mathrm{O}-\beta \mathrm{R}$ & 3 & 4 & 5 & 84.85 & 85.41 & 86.52 & 86.59 & 86.81 & 84.95 \\
\hline 20 & Naringin & 3 & 4 & 5 & 105.43 & 101.18 & 100.35 & 102.77 & 102.83 & 95.58 \\
\hline 21 & Hesperidin & 3 & 4 & 5 & 94.34 & 92.91 & 93.21 & 104.43 & 103.58 & 96.13 \\
\hline 22 & Daidzein & 3 & 4 & 5 & 92.03 & 91.27 & 92.08 & 89.38 & 92.19 & 87.70 \\
\hline 23 & Trans-cinnamic acid & 3 & 4 & 5 & 105.93 & 102.18 & 100.70 & 103.32 & 103.03 & 95.92 \\
\hline 24 & Quercetin dihydrate & 3 & 4 & 5 & 88.30 & 88.62 & 90.79 & 84.89 & 89.65 & 84.86 \\
\hline
\end{tabular}


Table 4. Cont.

\begin{tabular}{cccccccccccc}
\hline \multirow{2}{*}{ Sr. No. } & \multirow{2}{*}{ Compound } & \multicolumn{3}{c}{ Spiked Amount $(\mu \mathrm{g} / \mathbf{m L})$} & \multicolumn{3}{c}{ Recovery \% (Sample C) } & \multicolumn{3}{c}{ Recovery \% (Sample D) } \\
\cline { 3 - 12 } & & $\mathbf{7 5 \%}$ & $\mathbf{1 0 0} \%$ & $\mathbf{1 2 5} \%$ & $\mathbf{7 5 \%}$ & $\mathbf{1 0 0} \%$ & $\mathbf{1 2 5} \%$ & $\mathbf{7 5 \%}$ & $\mathbf{1 0 0 \%}$ & $\mathbf{1 2 5 \%}$ \\
\hline 25 & Naringenin & 3 & 4 & 5 & 91.58 & 91.32 & 92.10 & 88.73 & 92.23 & 87.64 \\
26 & Apigenin & 3 & 4 & 5 & 108.33 & 104.63 & 103.17 & 104.91 & 104.71 & 98.04 \\
27 & Kaempferol & 3 & 4 & 5 & 105.96 & 102.63 & 101.07 & 103.24 & 103.18 & 96.52 \\
28 & 2-MC & 3 & 4 & 5 & 90.95 & 91.14 & 92.41 & 88.17 & 91.69 & 87.12 \\
\hline
\end{tabular}

Table 5. Precision of the method.

\begin{tabular}{|c|c|c|c|c|c|c|c|c|c|}
\hline \multirow{3}{*}{ Sr. No. } & \multirow{3}{*}{ Compound } & \multicolumn{4}{|c|}{ Precision } & \multicolumn{4}{|c|}{ Intermediate Method Precision } \\
\hline & & \multicolumn{2}{|c|}{ Spiked Sample C } & \multicolumn{2}{|c|}{ Spiked Sample D } & \multicolumn{2}{|c|}{ Spiked Sample C } & \multicolumn{2}{|c|}{ Spiked Sample D } \\
\hline & & $\begin{array}{c}\text { RT } \\
(\% \text { RSD) }\end{array}$ & $\begin{array}{c}\text { AREA } \\
\text { (\%RSD) }\end{array}$ & $\begin{array}{c}\text { RT } \\
\text { (\%RSD) }\end{array}$ & $\begin{array}{c}\text { AREA } \\
\text { (\%RSD) }\end{array}$ & $\begin{array}{c}\text { RT } \\
\text { (\%RSD) }\end{array}$ & $\begin{array}{c}\text { AREA } \\
\text { (\%RSD) }\end{array}$ & $\begin{array}{c}\text { RT } \\
\text { (\%RSD) }\end{array}$ & $\begin{array}{c}\text { AREA } \\
\text { (\%RSD) }\end{array}$ \\
\hline 1 & Gallic acid & 0.4 & 0.58 & 0.1 & 3.19 & 0.3 & 2.05 & 0.3 & 4.0 \\
\hline 2 & 3,5 DHBA & 0.3 & 0.46 & 0.0 & 1.12 & 0.3 & 0.75 & 0.4 & 2.38 \\
\hline 3 & 3,4 DHBA & 0.3 & 0.74 & 0.0 & 1.25 & 0.3 & 0.82 & 0.4 & 1.09 \\
\hline 4 & Catechol & 0.2 & 0.68 & 0.1 & 0.83 & 0.2 & 1.05 & 0.3 & 1.15 \\
\hline 5 & Chlorogenic acid & 0.2 & 0.75 & 0.2 & 0.95 & 0.4 & 1.33 & 0.5 & 1.84 \\
\hline 6 & Catechin & 0.2 & 0.73 & 0.2 & 1.62 & 0.4 & 1.01 & 0.5 & 2.09 \\
\hline 7 & Caffeine & 0.2 & 0.12 & 0.2 & 1.20 & 0.4 & 0.56 & 0.5 & 1.26 \\
\hline 8 & Syringic acid & 0.2 & 0.51 & 0.1 & 0.62 & 0.4 & 0.49 & 0.4 & 1.04 \\
\hline 9 & Procyanidin B2 & 0.2 & 2.96 & 0.2 & 1.99 & 0.4 & 2.42 & 0.4 & 2.07 \\
\hline 10 & Vanillin & 0.1 & 0.14 & 0.1 & 1.34 & 0.3 & 0.5 & 0.3 & 1.27 \\
\hline 11 & Trans- $p$-coumaric acid & 0.1 & 0.69 & 0.1 & 0.81 & 0.3 & 0.58 & 0.3 & 1.34 \\
\hline 12 & Salicylic acid & 0.1 & 1.89 & 0.1 & 4.09 & 0.2 & 1.58 & 0.3 & 3.06 \\
\hline 13 & Trans-ferulic acid & 0.1 & 0.57 & 0.1 & 0.97 & 0.2 & 0.40 & 0.2 & 1.22 \\
\hline 14 & Trans-sinapic acid & 0.1 & 0.89 & 0.1 & 0.75 & 0.2 & 1.35 & 0.3 & 1.97 \\
\hline 15 & Taxifolin & 0.1 & 0.38 & 0.1 & 1.25 & 0.2 & 0.33 & 0.3 & 1.34 \\
\hline 16 & Ellagic acid & 0.1 & 1.35 & 0.1 & 2.21 & 0.2 & 1.31 & 0.3 & 1.94 \\
\hline 17 & Rutin hydrate & 0.1 & 2.38 & 0.1 & 2.34 & 0.2 & 1.63 & 0.3 & 2.33 \\
\hline 18 & Isoquercetin & 0.1 & 0.91 & 0.0 & 1.95 & 0.2 & 1.10 & 0.3 & 1.91 \\
\hline 19 & $\mathrm{~K}-3-\mathrm{O}-\beta \mathrm{R}$ & 0.1 & 1.14 & 0.0 & 1.11 & 0.2 & 0.93 & 0.2 & 1.19 \\
\hline 20 & Naringin & 0.1 & 0.35 & 0.0 & 0.80 & 0.1 & 0.74 & 0.1 & 1.96 \\
\hline 21 & Hesperidin & 0.1 & 0.54 & 0.0 & 1.20 & 0.1 & 0.49 & 0.1 & 1.25 \\
\hline 22 & Daidzein & 0.0 & 0.26 & 0.0 & 1.44 & 0.1 & 1.10 & 0.1 & 1.62 \\
\hline 23 & Trans-cinnamic acid & 0.0 & 0.17 & 0.0 & 1.04 & 0.1 & 0.32 & 0.1 & 1.19 \\
\hline 24 & Quercetin dehydrate & 0.0 & 0.89 & 0.0 & 1.47 & 0.1 & 0.96 & 0.1 & 1.94 \\
\hline 25 & Naringenin & 0.0 & 0.23 & 0.0 & 1.29 & 0.1 & 0.67 & 0.0 & 1.43 \\
\hline 26 & Apigenin & 0.0 & 0.15 & 0.0 & 1.16 & 0.0 & 0.46 & 0.0 & 1.33 \\
\hline 27 & Kaempferol & 0.0 & 0.21 & 0.0 & 0.92 & 0.0 & 0.36 & 0.0 & 1.04 \\
\hline 28 & 2-MC & 0.0 & 0.81 & 0.0 & 1.76 & 0.0 & 0.93 & 0.0 & 1.46 \\
\hline
\end{tabular}

\subsubsection{Application of Method}

To test the feasibility of developed method, leaves of healthy cotton plant and roots of diseased (root rot) (sample C) and healthy (sample D) plants were analyzed for phenolic compounds. Catechol and caffeine were exclusively found in the diseased root sample. The diseased plant possessed significantly higher content of all quantified phenolic compounds except 2- methoxycinnamaldehyde which is higher in healthy root sample (Figures 3 and 4). The highest concentration of rutin hydrate $(22.05 \mu \mathrm{g} / \mathrm{g})$ followed by isoquercetin $(7.05 \mu \mathrm{g} / \mathrm{g})$ was found in healthy leaf sample (Figure 5). Results of the study emphasizes that the developed procedure allows excellent resolution and sensitivity which further enable the estimation of very small amounts of phenolics in the sample. 


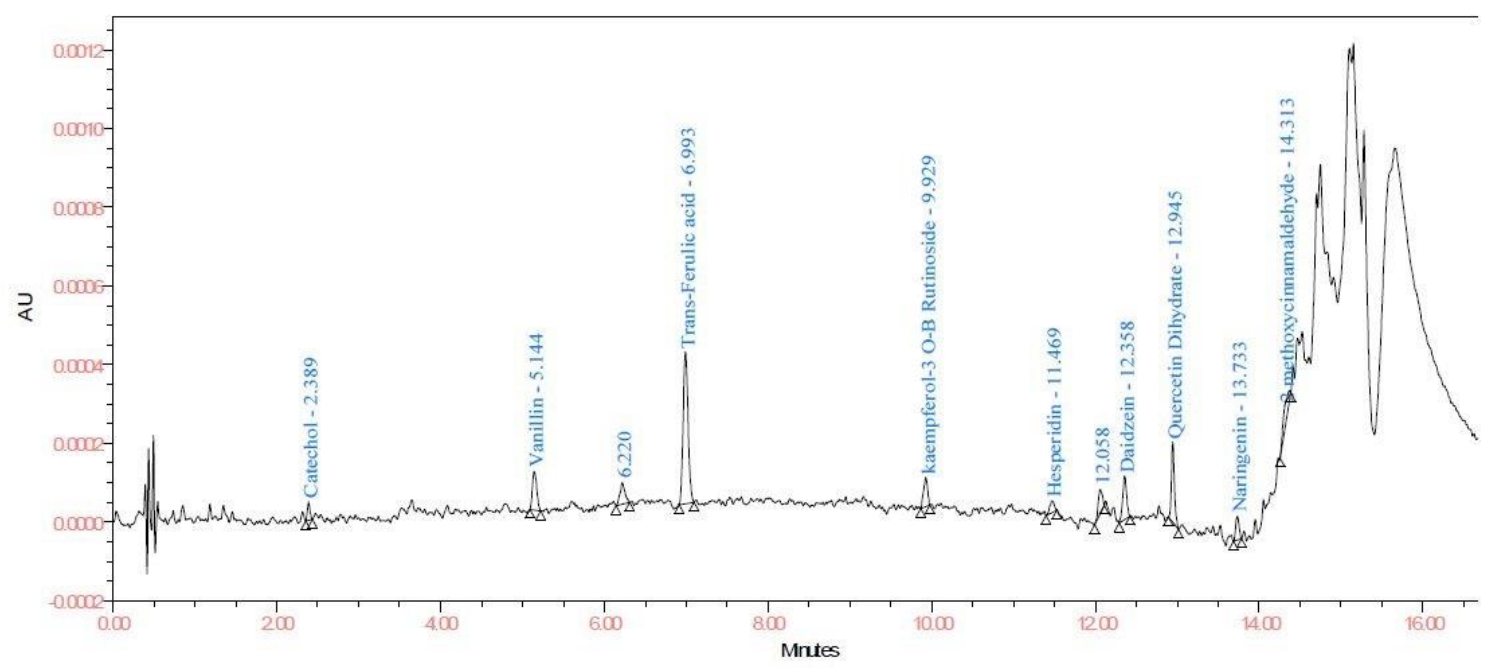

Figure 3. Chromatogram of diseased cotton root sample (sample C) at $278 \mathrm{~nm}$.

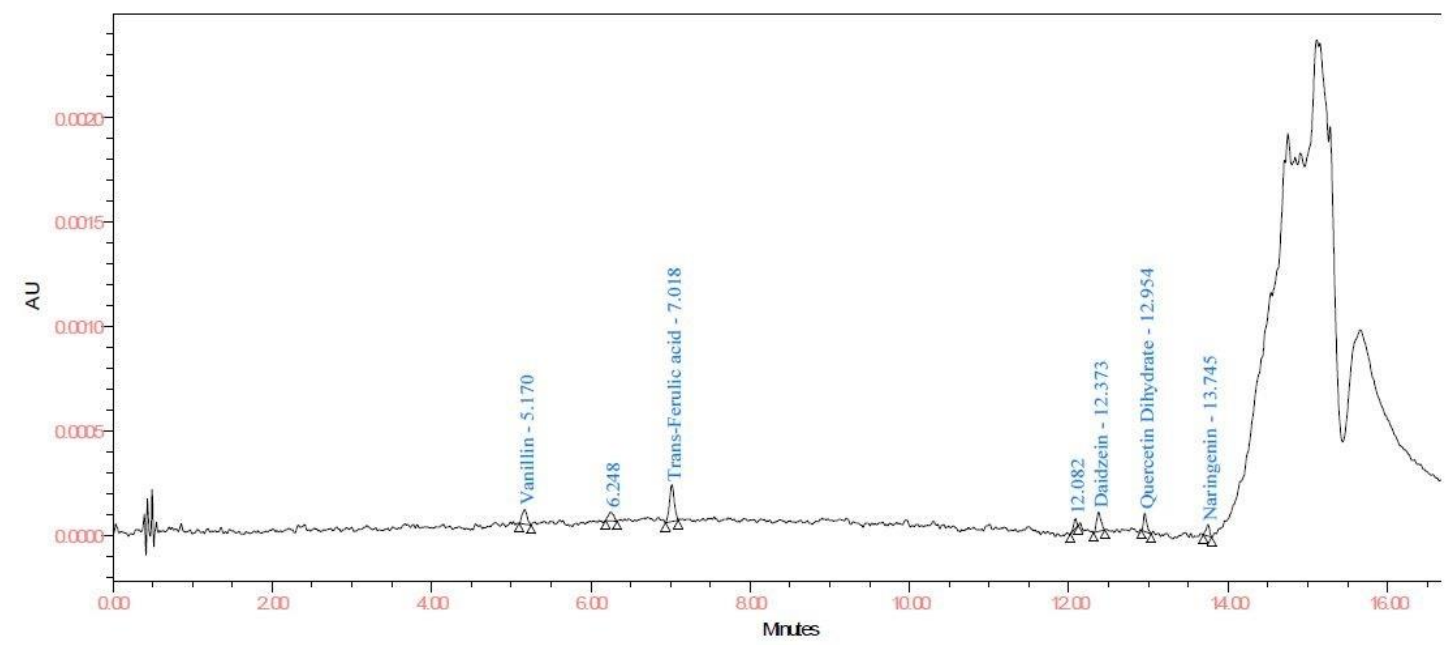

Figure 4. Chromatogram of cotton root sample (sample D) at $278 \mathrm{~nm}$.

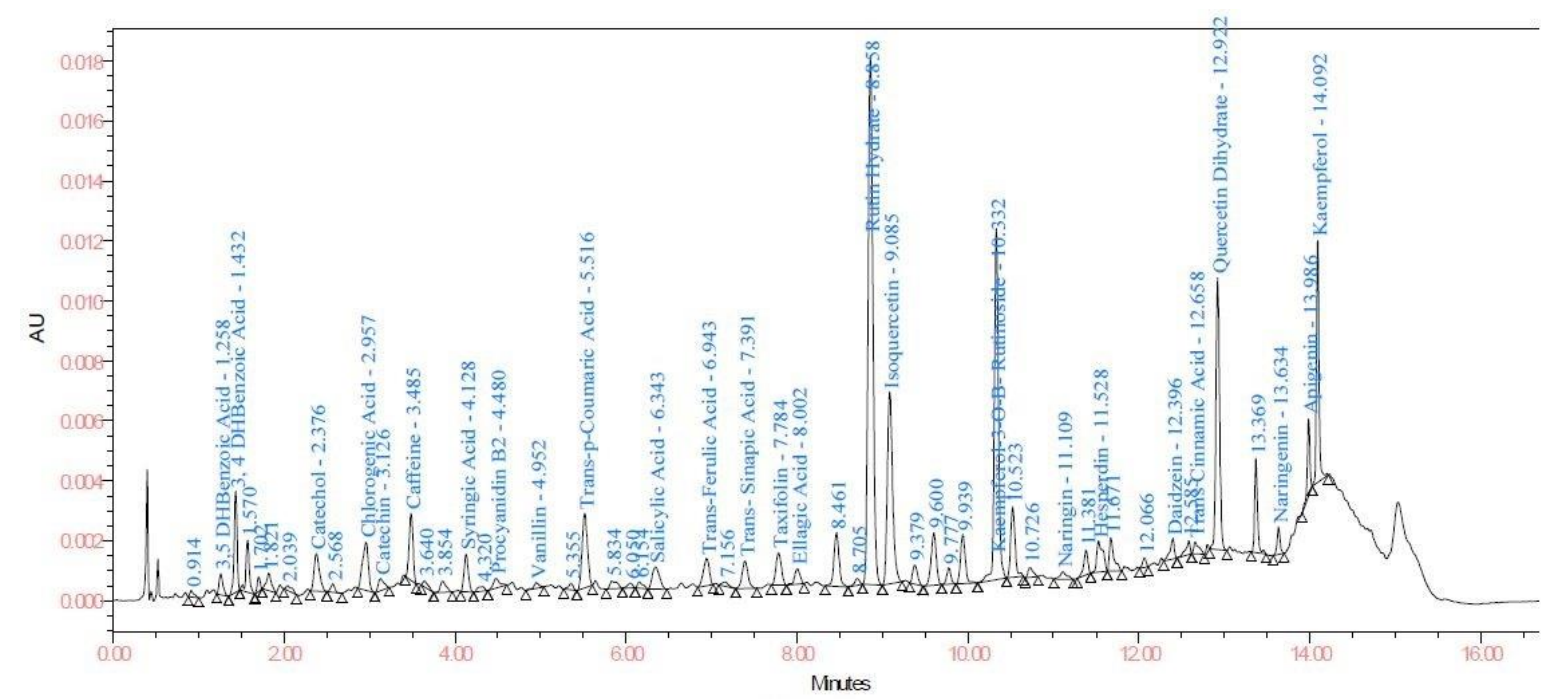

Figure 5. Chromatogram of healthy cotton leaf sample at $278 \mathrm{~nm}$. 


\section{Conclusions}

The present study describes the development of a model for simultaneous resolution and quantification of 28 phenolic compounds including isobaric species $(3,4$ and 3,5 dihydroxybenzoic acid; taxifolin and quercetin) with high accuracy and sensitivity using a UPLC-PDA system. The developed method was successfully applied in cotton leaves and roots to quantify the levels of various phenolic compounds. Findings of the study provide an option for swift and efficient quantification of plant phenolics at low cost.

Supplementary Materials: The following are available online at http://www.mdpi.com/2218-273X/10/1/6/s1, The figures A(1-3) Chromatograms of peak purity: 1- Peak purities of standards, 2- Peak purities of spiked sample C, 3- Peak purities of spiked sample D,B (1-28) Linear curves obtained through Empower 3 software at 278nm: 1- Gallic acid, 2- 3,5 Dihydroxy benzoic acid, 3-3,4 Dihydroxybenzoic acid, 4-Catechol, 5-Chlorogenic acid, 6-Catechin, 7-Caffeine, 8-Syringic acid, 9-Procyanidin B2, 10-Vanillin, 11- Trans-coumaric acid, 12-Salicylic acid, 13-Trans-ferulic acid, 14-Trans-sinapic acid, 15-Taxifolin, 16-Ellagic acid, 17-Rutin hydrate, 18-Isoquercetin, 19-Kaempferol-3-O-ßrutinoside, 20-Naringin, 21-Hesperdin, 22-Daidzein, 23-Trans-cinnamic acid, 24-Quercetin dihydrate, 25-Naringenin, 26-Apigenin, 27-Kaempferol, 28-2-Methoxycinnmaldehyde and C (1-2). Chromatogram of LOD (C.1), LOQ (C.2) obtained at 278nm.

Author Contributions: Conceptualization, methodology, investigation, formal analysis, supervision, writing original draft, S.M.; review and editing, A.P.; visualization, V.S. All authors have read and agreed to the published version of the manuscript.

Funding: This research did not welcome any specific grant from funding agencies in the public, commercial, or not-for-profit sectors.

Acknowledgments: The author is very grateful to Yogesh Sharma, Waters India Pvt Ltd, New Delhi, India for technical assistance in the execution of this method. The author is also thankful to the Scientists of Cotton Section, Department of Genetics and Plant Breeding, CCS Haryana Agricultural University, Hisar, India for their moral supports.

Conflicts of Interest: The authors declare that there is no conflict of interest.

\section{References}

1. Lee, J.J.; Woodward, A.W.; Chen, Z.J. Gene expression changes and early events in cotton fibre development. Ann. Bot. 2007, 100, 1391-1401. [CrossRef] [PubMed]

2. Wendel, J.F.; Cronn, R.C. Polyploidy and the evolutionary history of cotton. Adv. Agron. 2003, 78, 139-186.

3. Razaq, M.; Aslam, M.; Shad, S.A.; Aslam, M.N.; Saeed, N.A. Evaluation of some new promising cotton strains against bollworm complex. J. Res. Sci. 2004, 15, 313-318.

4. Dua, I.S.; Kumar, V.; Bhavneet, D.E.A. Genetically modified cotton and its Biosafety concerns: A review. In Current Concepts in Botany (Book), 1st ed.; Mukerji, K.G., Manoharachary, C., Eds.; I.K. International Publishing House Pvt. Ltd.: New Delhi, India, 2006; pp. 447-459.

5. Lawo, N.C.; Wackers, F.L.; Romeis, J. Indian Bt cotton varieties do not affect the performance of cotton aphids. PLoS ONE 2009, 4, e4804. [CrossRef] [PubMed]

6. Satpute, U.S.; Patil, V.N.; Katole, S.R.; Men, U.B.; Bhagwat, V.R.; Thakare, A.Y. Avoidable field losses due to sucking pests and bollworms in cotton. J. Appl. Zool. Res. 1990, 1, 67-72.

7. Kempema, L.A.; Cui, X.; Holzer, F.M.; Walling, L.L. Arabidopsis transcriptome changes in response to phloem-feeding Silverleaf whitefly nymphs. Similarities and distinctions in responses to aphids. Plant Physiol. 2007, 143, 849-865. [CrossRef] [PubMed]

8. Shalash, M.; Makahleh, A.; Salhimi, S.M.; Saad, B. Vortex-assisted liquid-liquid-liquid microextraction followed by high performance liquid chromatography for the simultaneous determination of fourteen phenolic acids in honey, iced tea and canned coffee drinks. Talanta 2017, 174, 428-435. [CrossRef]

9. Association of Official Analytical Chemists, Official Methods of the Association of the Agricultural Chemists, eighteen Ed., Gaithersburg, 2007

10. Peled-Zehavi, H.; Oliva, M.; Xie, Q.; Tzin, V.; Oren-Shamir, M.; Aharoni, A.; Galili, G. Metabolic engineering of the phenylpropanoid and its primary, precursor pathway to enhance the flavor of fruits and the aroma of flowers. Bioengineering (Basel) 2015, 2, 204-212. [CrossRef] 
11. Cocuron, J.C.; Casas, M.I.; Yang, F.; Grotewold, E.; Alonso, A.P. Beyond the wall: High-throughput quantification of plant soluble and cell-wall bound phenolics by liquid chromatography tandem mass spectrometry. J. Chromatogr. A 2019, 1589, 93-104. [CrossRef]

12. Lege, K.E.; Cothren, J.T.; Smith, C.W. Phenolic acid and condensed tannin concentrations of six cotton genotypes. Environ. Exp. Bot. 1995, 35, 241-249. [CrossRef]

13. Liu, Q.; Cai, W.; Shao, X. Determination of seven polyphenols in water by high performance liquid chromatography combined with preconcentration. Talanta 2008, 77, 679-683. [CrossRef]

14. Naczk, M.; Shahidi, F. Review: Extraction and analysis of phenolics in food. J. Chromatogr. A 2004, 1054, 95-111. [CrossRef]

15. Khoddami, A.; Wilkes, M.A.; Roberts, T.H. Techniques for analysis of plant phenolic compounds. Molecules 2013, 18, 2328-2375. [CrossRef]

16. Mattila, P.; Kumpulainen, J. Determination of free and total phenolic acids in plant-derived foods by HPLC with diode-array detection. J. Agric. Food Chem. 2002, 50, 3660-3667. [CrossRef]

17. Santiago, R.; Lopez-Malvar, A.; Souto, C.; Barros-Rios, J. Methods for determining cell wall-bound phenolics in maize stem tissues. J. Agric. Food Chem. 2018, 66, 1279-1284. [CrossRef]

18. Garzon, G.A.; Narvaez-Cuenca, C.E.; Vincken, J.P.; Gruppen, H. Polyphenolic composition and antioxidant activity of Acai (Euterpe oleracea Mart.) from Colombia. Food Chem. 2017, 217, 364-372. [CrossRef] [PubMed]

19. Ramirez-Ambrosi, M.; Abad-Garcia, B.; Viloria-Bernal, M.; Garmon-Lobato, S.; Berrueta, L.A.; Gallo, B. A new ultra high performance liquid chromatography with diode array detection coupled to electrospray ionization and quadrupole time-of-flight mass spectrometry analytical strategy for fast analysis and improved characterization of phenolic compounds in apple products. J. Chromatogr. A 2013, 1316, 78-91. [PubMed]

20. Simirgiotis, M.J.; Quispe, C.; Borquez, J.; Areche, C.; Sepulveda, B. Fast detection of phenolic compounds in extracts of easter pears (Pyrus communis) from the Atacama desert by ultrahigh-performance liquid chromatography and mass spectrometry (UHPLC-Q/Orbitrap/MS/MS). Molecules 2016, 21, 92. [CrossRef] [PubMed]

21. Vagiri, M.; Ekholm, A.; Andersson, S.C.; Johansson, E.; Rumpunen, K. An optimized method for analysis of phenolic compounds in buds, leaves, and fruits of black currant (Ribes nigrum L.). J. Agric. Food Chem. 2012, 60, 10501-10510. [CrossRef]

22. Jaini, R.; Wang, P.; Dudareva, N.; Chapple, C.; Morgan, J.A. Targeted metabolomics of the phenylpropanoid pathway in Arabidopsis thaliana using reversed phase liquid chromatography coupled with tandem mass spectrometry. Phytochem. Anal. 2017, 28, 267-276. [CrossRef]

23. Casas, M.I.; Duarte, S.; Doseff, A.I.; Grotewold, E. Flavone-rich maize: an opportunity to improve the nutritional value of an important commodity crop. Front. Plant. Sci. 2014, 5, 440. [CrossRef] [PubMed]

24. Orcic, D.; Franciskovic, M.; Bekvalac, K.; Svircev, E.; Beara, I.; Lesjak, M.; Mimica-Dukic, N. Quantitative determination of plant phenolics in Urticadioica extracts by high-performance liquid chromatography coupled with tandem mass spectrometric detection. Food Chem. 2014, 143, 48-53. [CrossRef] [PubMed]

25. Vieira, G.S.; Marques, A.S.F.; Machado, M.T.C.; Silva, V.M.; Hubinger, M.D. Determination of anthocyanins and non-anthocyanin polyphenols by ultra performance liquid chromatography/electrospray ionization mass spectrometry (UPLC/ESI-MS) in Jussara (Euterpe edulis) extracts. J. Food Sci. Technol. 2017, 54, 2135-2144. [CrossRef] [PubMed]

26. Adom, K.K.; Liu, R.H. Antioxidant activity of grains. J. Agric. Food Chem. 2002, 50, 6182-6187. [CrossRef] [PubMed]

27. Irakli, M.N.; Samanidou, V.F.; Biliaderis, C.G.; Papadoyannis, I.N. Simultaneous determination of phenolic acids and flavonoids in rice using solid-phase extraction and RP-HPLC with photodiode array detection. J. Sep. Sci. 2012, 35, 1603-1611. [CrossRef] [PubMed]

28. Amarowicz, R.; Weidner, S. Content of phenolic acids in rye caryopses determined using DAD-HPLC method. Czech. J. Food Sci. 2001, 19, 201-205. [CrossRef]

29. Fracassetti, D.; Lawrence, N.; Tredoux, A.G.J.; Tirelli, A.; Nieuwoudt, H.H.; Du Toit, W.J. Quantification of glutathione, catechin and caffeic acid in grape juice and wine by a novel ultra-performance liquid chromatography method. Food Chem. 2011, 128, 1136-1142. [CrossRef]

30. Dias, F.S.; Lovillo, M.P.; Barroso, C.G.; David, J.M. Optimization and validation of a method for the direct determination of catechin and epicatechin in red wines by HPLC/fluorescence. Microchem. J. 2010, 96, 17-20. [CrossRef] 
31. Escarpa, A.; González, M.C. Optimization strategy and validation of one chromatographic method as approach to determine the phenolic compounds from different sources. J. Chromatogr. A 2000, 897, 161-170. [CrossRef]

32. SemanticScholar. Available online: https://pdfs.semanticscholar.org/5d48/ 0d7b3e906131b2cb5eba3c2cc87192cb78df.pdf?_ga=2.122522187.1122837214.1576659057-437361496. 1575990593 (accessed on 16 November 2019).

(c) (C) 2019 by the authors. Licensee MDPI, Basel, Switzerland. This article is an open access article distributed under the terms and conditions of the Creative Commons Attribution (CC BY) license (http://creativecommons.org/licenses/by/4.0/). 